\title{
Cooperative Object Transfer: Effect of Observing Different Part of the Object on the Cooperative Task Smoothness*
}

\author{
Ahmad Faizal SALLEH**, Ryojun IKEURA**, Soichiro HAYAKAWA** \\ and Hideki SAWAI** \\ ${ }^{* *}$ Division of Mechanical Engineering, Graduate School of Engineering, Mie University \\ Kurimamachiyacho 1577, Tsu, Mie 514-8507, Japan \\ E-mail: faizal@ss.mach.mie-u.ac.jp
}

\begin{abstract}
Designing robots with human characteristics is a great challenge to researcher to fulfill the needs of robotic technology in human occupied environment. This research aims to enable a smooth and natural cooperative object transfer by a human hand and a robot manipulator mimicking the same task performed by two humans. Prior to the development of the human-robot system, investigation on characteristics which generate a smooth cooperative object transfer in a human-human system is crucial. Perceiving certain part of an object in transfer, acts as a medium for the exchange of object's motion information between subjects. We have investigated the effect of perceiving different parts of the object in transfer to the cooperative motion smoothness (smoothness is quantitatively evaluated using Minimum Jerk Model). The result suggests that observing the center part of the experimental object produced more frequent, a smooth and natural motion for task executed in leftward/rightward and upward/downward direction. Moreover, the center part was associated with less object rotation during smooth task in these directions. However, there is no significant difference between End and Center case for the same task executed in forward/backward direction. We also considered the importance of having information of cooperative task initiation signal and a target position to the cooperative task smoothness. Regardless of which part of the object being perceived, smoother task is frequently generated when both signals are available to both subjects. Although the effect of End and Center case may be significant in human-human system; in human-robot system, both cases should be further tested and evaluated.
\end{abstract}

Key words: Cooperative Object Transfer, Cooperative Task Smoothness, Minimum Jerk Model, Information Exchange, Human-Human Interaction

\section{Introduction}

The necessity of utilizing a robot as a partner during cooperative task is increasing due to some factors (shortage of human labor, power assistance, cost reduction and etc). One of the example is the cooperative task of transferring an object from one position to another involving both hand and manipulator. The manipulator motion mimicking human hand motion is important to enable a comfortable cooperation between human and robot.

Some researches show that communication is important during cooperative motion of two agents ${ }^{(1)-(4)}$. Markus et al ${ }^{(5)}$ has investigated the hand-over task of a common object between two humans and compared the results with human-robot system. Two velocity

${ }^{*}$ Received 12 Apr., 2011 (No. 11-0245) [DOI: 10.1299/jbse.6.343]

Copyright (C) 2011 by JSME 
profiles i.e. a conventional trapezoidal and a minimum jerk profile was utilized in their human-robot system. They found better results with the minimum jerk profile. In Ref. (6), the cooperative transfer of a long object in a vertical direction by two humans was studied. The importance of the object posture information to the slave was reported. Another study on the cooperative object transfer in human-human system was reported ${ }^{(7)}$. A handled shape object was transferred to a final position which located at a distance and at the same height of the initial position. They found a better model to represent the two dimensional cooperative motions. However, most of the reported studies did not account on perceiving the object motion as a means of communication for a smooth cooperative transfer.

Our main objective is to obtain a smooth and natural cooperative rigid body transfer between human hand and robot manipulator mimicking the same task executed by two humans. Thus, investigating the characteristics that generate smooth and natural cooperative transfer in human-human system is crucial. In this article, we compare the effect of perceiving at different or same part of the image (this image represent the real object in transfer) as a communication means for both subjects during the cooperative task. We hypothesized that if during the task, both subjects perceive (communicate) at the same part of the image, they will have similar object motion information to move from the start to the target position. Thus, smoother cooperative motion is expected. In contrary, when they perceive at different part of the image during transfer, they will not have similar object motion information during the task. Therefore, we conducted an experiment where subjects were asked to perceive the image with two different ways, i.e. by looking at the end or at the center part (henceforth referred to as End and Center, respectively) of the image. The End case refers to the condition where both subjects perceive at two different part of the image and did not posses similar object motion information. The Centre case refers to the case where both subjects perceive at the same part of the image and posses similar object motion information during the task.

A similar investigation was carried out in previous research ${ }^{(8)}$; however, they only focus on the End case. Furthermore, an error value which is the difference between actual velocity and minimum jerk velocity was utilized as a smoothness indicator. In our research, instead of using the error value ${ }^{(8)}$, the jerk value was utilized as the smoothness indicator. The jerk value indicates more accurate motion smoothness since it is the third derivative of position over time. Additionally, we also hypothesized that less object rotation is expected during smooth cooperative task. Therefore, we compare the effect of End and Center case on the object rotation during smooth cooperative task. The hand movement towards target consisted of two motion phases ${ }^{(9)}$ i.e. the transfer phase from start to target position and the precise positioning phase at target. In this research, the precise positioning phase was excluded. This was done to resemble the natural hand motion in a non-precise positioning cooperative task (e.g. carrying task at home, office, etc). The next section of this paper discusses about the Minimum Jerk Model (MJM) as a smoothness indicator for cooperative motion. The third section will elaborate on the experimental research procedures as well as the equipment used. The remainder of the paper is devoted to discussion on the analyzed data.

\section{Mathematical Model as Smoothness Indicator}

The MJM is utilized to analyze and evaluate cooperative motion smoothness quantitatively. The model adapts the optimal control methodology which has been proven to be the smoothest movement of human arm that can be achieved by minimizing jerk during the motion ${ }^{(10)}$. Jerk is defined as the change of rate of acceleration; thus, it is a third temporal derivative of position. In other words, the model can be used to predict the kinematics aspect of smooth human arm motion which includes the arm position, velocity, 
acceleration and jerk. Assuming that $x$ and $y$ are the time varying hand positions in Cartesian coordinate, the magnitude of the jerk to be minimized can be shown as,

$$
\text { Jerk }=\sqrt{\left(\frac{d^{3} x}{d t^{3}}\right)^{2}+\left(\frac{d^{3} y}{d t^{3}}\right)^{2}}
$$

In moving a human hand from an initial to a final position in a given time $t_{f}$, the cost function $C$ to be minimized is the time integral of the square of the magnitude of jerk:

$$
C=\frac{1}{2} \int_{0}^{t_{f}}\left[\left(\frac{\mathrm{d}^{3} x}{\mathrm{~d} t^{3}}\right)^{2}+\left(\frac{\mathrm{d}^{3} y}{\mathrm{~d} t^{3}}\right)^{2}\right] \mathrm{d} t
$$

In our experiment, a one dimensional movement is considered; therefore the above minimum jerk formula can be simplified as,

$$
C=\frac{1}{2} \int_{0}^{t_{f}}\left[\left(\frac{\mathrm{d}^{3} x}{\mathrm{~d} t^{3}}\right)^{2}\right] \mathrm{d} t
$$

Although the MJM is used for a single human operator, in our case, we assume that for a smooth cooperative motion, one subject will follow another subject movement while maintaining his/her own jerk at minimum level. Based on this concept, the MJM is used in our experiment to evaluate the smoothness of human-human cooperative tasks quantitatively. The investigation on the object rotational motion is based on the following formula,

$$
\theta_{\text {ave }}=\frac{1}{t_{f}} \int_{0}^{t_{f}} a b s(\theta(t)) \mathrm{d} t
$$

where $\theta(t)$ is the instantaneous angle of the object during the cooperative task

\section{Method}

\subsection{Experimental Devices}

The experimental equipment consists of a position measurement system (Optotrack Certus 3020 camera, System Control Unit and Personal Computer), two monitor screens and a rigid experimental object as shown in Fig. 1. Figure 2 shows the experimental object dimensions and configuration. The dimensions of the experimental object are $60 \mathrm{~mm}(\mathrm{H}) \mathrm{x}$ $180 \mathrm{~mm}(\mathrm{~W}) \times 460 \mathrm{~mm}(\mathrm{~L})$ and weighed about $3 \mathrm{~kg}$. It is equipped with small infrared light emitting diode (IR LED) markers on its sides. When both subjects moved the experimental object in any directions, a three dimensional (3D) camera in the position measurement system detected signal from the diode marker and sent it to the main computer. The computer processed the signal and converted it to a two dimensional (2D) rectangular image mimicking the experimental object's motion in real time. The rectangular image size is 10 $\mathrm{mm}(\mathrm{W}) \times 100 \mathrm{~mm}(\mathrm{~L})$. In leftward/rightward task, the image $(10 \mathrm{~mm} \times 100 \mathrm{~mm})$ represents $180 \mathrm{~mm} \times 460 \mathrm{~mm}$ surface area of the real object. In upward/downward and forward/backward task, the image $(10 \mathrm{~mm}$ X $100 \mathrm{~mm}$ ) represents $60 \mathrm{~mm}$ x $460 \mathrm{~mm}$ surface area of the real object. The image was displayed on the monitors screen in front of both subjects. The screen was provided as a visual assistance device for subjects to perceive the object clearly and also as reference of motion ${ }^{(4)}$. Position data were recorded into the computer at every $10 \mathrm{~ms}$ of sampling interval. Position data from each marker was recorded 
based on the $\mathrm{X}, \mathrm{Y}$ and $\mathrm{Z}$ axis of the $3 \mathrm{D}$ camera. Data from each axis and marker were saved in a different columns with respect to the axis of the camera and marker (e.g. X, Y and Z data from marker 1 were saved in column 1, 2 and 3, and data from marker 2 were saved in column 4, 5 and 6 in matlab file). At the same time, the direction of the cooperative task was set based on the axis of the 3D camera. Positive $\mathrm{X}$ axis indicates the forward motion, negative $\mathrm{X}$ axis indicates the backward motion. In leftward/rightward task, positive $\mathrm{Z}$ refers to righward, while negative $\mathrm{Z}$ axis refers to leftward direction cooperative task. In upward/downward task, the positive $\mathrm{Y}$ axis indicates the upward task, while the negative $\mathrm{Y}$ axis indicates the downward task. When moving in one dimensional direction, the coordinates in that direction change more than the coordinate in the other directions. Using a matlab program, the distance of the cooperative task could be calculated. Therefore, the distance and direction could be confirmed during the analysis.
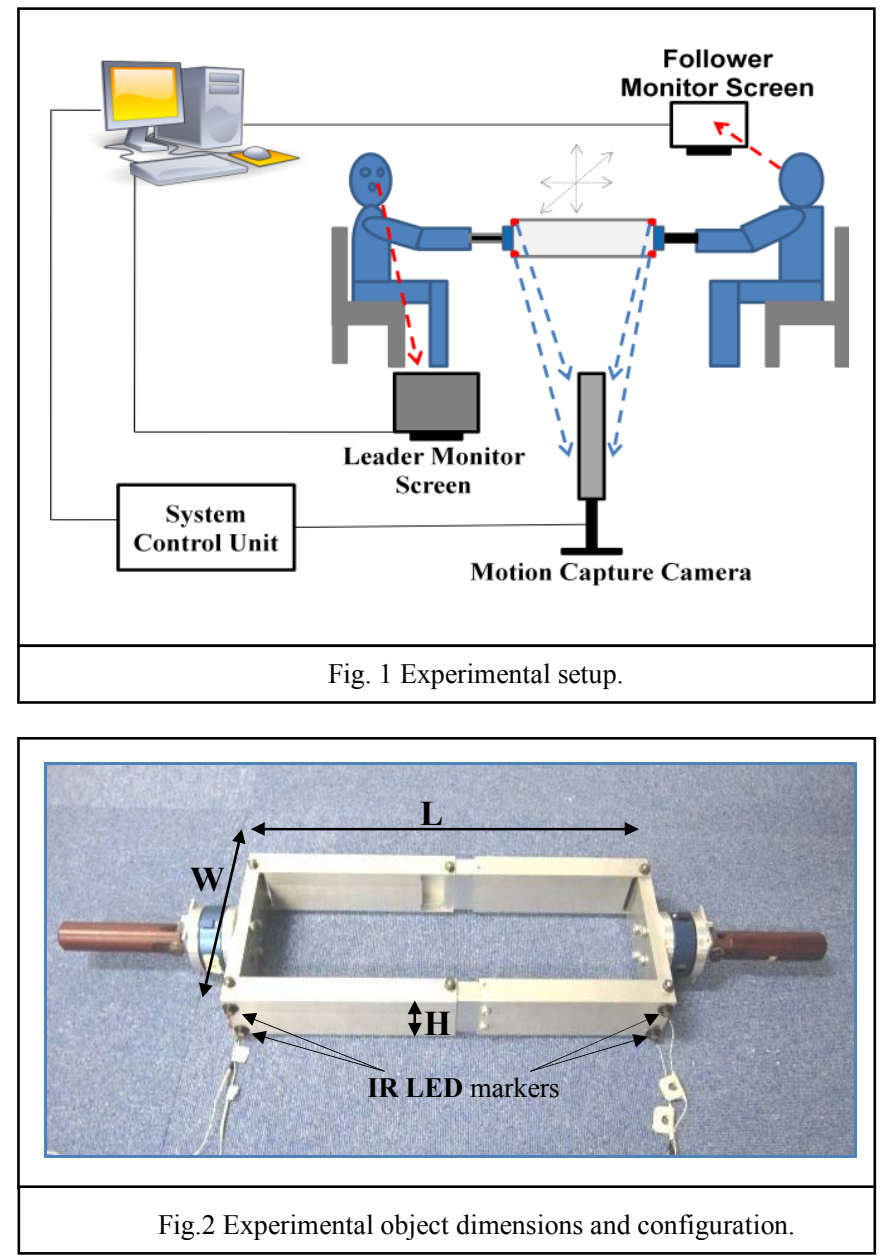

\subsection{Experimental Procedure}

The experiment was mainly designed to understand the effect of information exchange between humans in generating a smooth cooperative hand motion to transport a common object from one place to another in a seated position. We have selected 10 engineering students as experimental subjects, aged between 22 to 35 years old, who are physically and mentally healthy (no sensory, neurological, muscular, cutaneous or other impairment related problems). All of them have had no prior experiences in executing the current experiment. All subjects were provided with informed consents and instructions on the experiment. The 
subjects were classified into 5 groups, with each group consists of two persons. Only one group has a female participant, all the rest being males. In each group, one person was appointed as a Leader and the other as a Follower in executing the cooperative task. Both subjects were required to move the experimental object in upward/downward, leftward/rightward and forward/backward directions relative to the Leader. Mimicking the real cooperative task, the Leader initiated and decided the direction and target position of the task. Both of them started the task at the start position as shown in Fig. 3 (the start position in screen monitor is equivalent to the center position located between both subjects). Once received verbal instruction from the Leader, the Follower cooperated with the Leader in bringing the experimental object to the target position. Once the task was completed, both of them return the object to the start position. The 3D camera recorded their cooperative motion, then, the position data were saved in the computer. The targets were positioned at two distances in each direction namely, short and long distance. The short distance was set between 75 to $100 \mathrm{~mm}$ and long distance was between at 150 to 200 $\mathrm{mm}$ based on the subject suitability in each cooperative task direction. The subjects were required to practice moving the object for several times before they proceeded with the actual experiments.

Part of the information exchange is on how both subjects visualize and share the experimental object's motion information to enable a natural and smooth cooperative object transfer by hand ${ }^{(4)}$. Therefore, the experiment was designed to investigate the consequences of looking at different parts of the experimental object while matching it to the target position in generating a smooth cooperative motion. In the experiment, the subjects did not have direct visual contact on the experimental object but look at a $2 \mathrm{D}$ rectangular image resembling the experimental object translational and rotational motion in real time. The image was displayed on their respective screen monitors. Subjects were required to look at two different parts of the image, being the End or Center. Figure 3 shows the image perceived by subjects on screen monitors for cooperative task in the leftward/rightward, upward/downward and forward/backward direction.

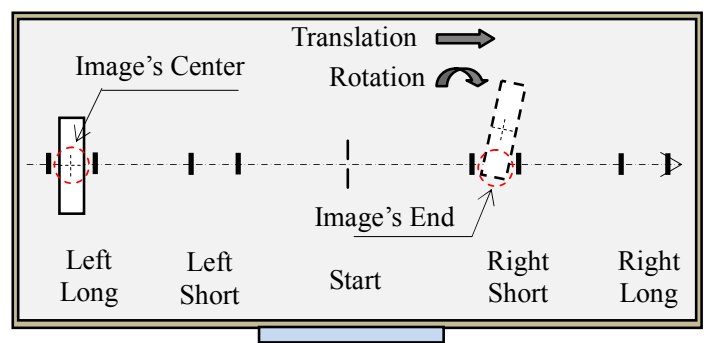

a)

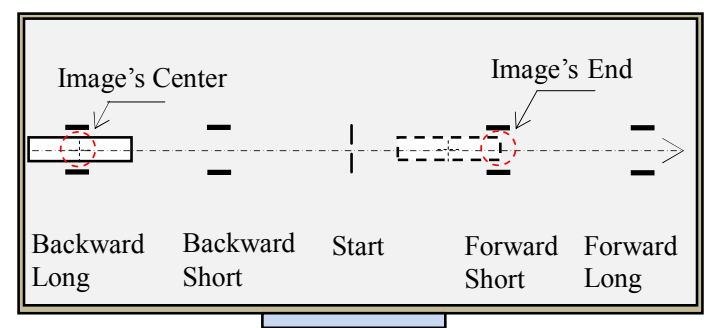

b)

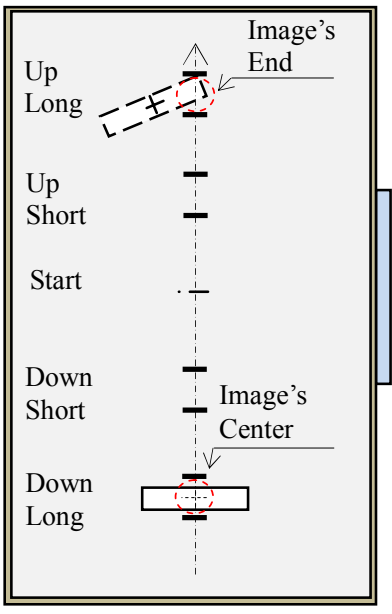

c)

Fig. 3 A 2D rectangular image displays on the screen monitors in front of both subjects for cooperative task in a) Leftward/rightward, b) Forward/backward, and c) Upward/downward direction. In any experiment, only one rectangular shaped image will be displayed on the screen (either image's Center or image's End).

First, we conducted the experiment with the End case for all groups of subjects and subsequently, proceeded to the Center case. It is very difficult for both subjects to position 
the experimental object's image to a precise target with the smoothness and naturalness of hand motion ${ }^{(10)}$. Therefore, the target was drawn as two parallel lines and if its center-line coincides with rectangular image's center-line, a $3 \mathrm{~mm}$ gap was provided to tolerate the image positioning. The targets increase the effectiveness of the cooperative task because it helps the Follower to know the exact termination point on the screen.

Another part of the information exchange was the effect of the Follower having explicit and/or implicit cooperative task information on the resulting motion smoothness ${ }^{(8)}$. The task information is verbal signal to initiate motion and the target position in order to terminate the cooperative task. There were cases when both information were explicitly given prior to the execution of the task by verbal means. In certain cases of the experiment, the information was not explicitly given to the Follower. However, based on vision (by looking at the rectangular image appears on the screen) and somatosensory (feeling the force acts on hand when holding the object), the Follower may predict the Leader intention to start and stop the cooperative motion. This is the meaning of implicit information possesses by the Follower during the task. Based on the combination of either giving/not the explicit information to the Follower, four modes of information delivery to the Follower was executed as shown in Table 1.

Table 1 Type of cooperative motion based on the information available to the Follower prior to the execution of the task

\begin{tabular}{|c|c|c|}
\hline Mode & $\begin{array}{c}\text { Explicit Information From the } \\
\text { Leader to the Follower }\end{array}$ & $\begin{array}{r}\text { Possible implicit information } \\
\text { utilized by the Follower to predict } \\
\text { and follow Leader's motion }\end{array}$ \\
\hline 1 & None & $\begin{array}{c}\text { Predict both Start and Stop- } \\
\text { Somatosensory, Visual. }\end{array}$ \\
\hline 2 & Starting Signal Only & Predict Stop - Somatosensory, Visual \\
\hline 3 & Target Position Only & Predict Start - Somatosensory, \\
Visual
\end{tabular}

On the signal to initiate the cooperative task; in mode 1, the Follower was not given any verbal instruction. However, in mode 2 , he/she was informed verbally to initiate the motion. The same procedure to initiate the cooperative task was carried out for modes 3 and 4 , respectively. On the target information to terminate the task, the Follower did not know the target position as for mode 1 and 2. Only, the Leader knew the target and selected them randomly to avoid the Follower predicting the desired stopping position. In contrast, in modes 3 and 4, both the Leader and the Follower have the target information and it is in an orderly manner to facilitate both subjects during the experiment. For example in leftward/rightward direction, the targets are in the order of left-short, left-long, right-short and finally right-long. Mode 1, 2 and 3 were lacked of explicit information, therefore the Follower was asked to follow the Leader motion by using the available implicit information, i.e by looking visually and feeling the object motion by hand. However, in mode 4, some test on the experiment had shown that following the Leader motion generated more awkward cooperative task. Moreover, previous researcher mentioned that the Follower moves towards target with his/her own rhythm (slightly difference rhythm from the Leader) and generated smoother cooperative task ${ }^{(8)}$. Thus, in mode 4 , the Follower was instructed not to follow the Leader motion. He/she will move the object towards the target position with his/her own rhythm once received instruction from the Leader.

In each mode, all subjects were required to move the experimental object in the leftward/rightward, upward/downward and forward/backward directions with two different 
distances as mentioned previously. The experiments were conducted with 10 repetitions for each direction and distance. Thus, about forty motions were recorded for each mode. Statistical analyses were performed using the Statistical Packages for the Social Sciences (SPSS). For each Mode the normalized mean jerk were computed to evaluate the cooperative motion smoothness. Differences between the levels of each variable were detected using repeated measure ANOVA. Significance level for all statistical tests was set at $p<0.01$. The measured position data were filtered with Butterworth filter at a cut-off frequency of $5 \mathrm{~Hz}$ programmed in Matlab software version $2007 \mathrm{~b}$.

\section{Result and Discussion}

\subsection{Kinematics profiles during cooperative task}

Figure 4 is the example of a smooth and an awkward cooperative task, in terms of position, velocity, acceleration and jerk profiles. The task was executed in leftward/rightward direction with a same distance and direction by the same subjects. The red and blue lines indicate the kinematics profiles based on the actual motion data of Leader and Follower, respectively. The green solid and dash-dot lines indicate the kinematics profiles based on the minimum jerk model of Leader and Follower, respectively. In each plot, the kinematics profiles based on the minimum jerk model (refers to green solid and dash-dot lines) has the same dimension as the actual motion profile (refers to red and blue line). As for example, the position profile based on minimum jerk model has the same dimension as actual position data, i.e. meter. This is also true for velocity, acceleration and jerk.

In smooth cooperative task (Fig. 4(a)), the actual motion and the minimum jerk profiles of each subject were almost similar. Each kinematics profiles were calculated based on the position data generated in the same direction of the cooperative task (i.e. moving leftward/rightward, the position data in leftward/rightward direction was used to calculate other profiles). The jerk profile shows peak value at the initiation, the middle and the termination point of the movement. Also, it was observed that the Follower had moved slightly faster than the Leader during the task. The data was obtained from mode 4, and it had been confirmed that the Follower moved faster than the Leader when he knew the target position ${ }^{(8)}$. Figures 4(b) and 4(c) show the kinematics profiles for the cooperative task of 4(a). However, the profiles in 4(b) and 4(c) were calculated based on the position data generated in the direction perpendicular to the cooperative task direction of 4(a) (i.e. moving leftward/rightward, using either forward/backward or upward/downward position data to calculate the other profiles). The kinematics profiles calculated in forward/backward direction (Fig. 4(b)) was smooth with a small jerk value. Follower's position profile (similar to Leader's profile) was not shown in Fig. 4(b) because it was in negative direction. In contrast, the kinematics profile calculated in upward/downward direction (Fig. 4(c)) was not so smooth. However, with a relatively small jerk value, the motion was considered smooth. Therefore, in one dimensional motion, using the jerk profile in the same direction of the cooperative task direction was sufficient to indicate the cooperative motion smoothness. In contrast to the smooth cooperative task (referring to 4(a)), in awkward task (Fig. 4(d), all profiles were calculated in the same direction of the cooperative task), such similarities were not found especially in the acceleration and jerk profiles. The jerk profile shows more fluctuation during the task execution.

Since jerk is the third temporal derivative of position, a long distance (also mean a longer time) task may produce more jerk than a short distance task. Thus, in the analysis, the actual jerk value was normalized with distance and time before it was used to calculate the minimum jerk value. By doing this, the effect of distance and time on the calculation of 
minimum jerk value was eliminated. Assume that $X$ is the normalized position, $x(t)$ is the actual position data at each sampling time, $t$. $t_{0}$ is the time at task initiation and $t_{f}$ is the time at task termination. The normalized position, normalized time, normalized jerk and normalized minimum jerk is shown in the following formulas,

Normalized Position, $X=\frac{x(t)}{x\left(t_{f}\right)-x\left(t_{0}\right)}$

Normalized Time, $T=\frac{t}{t_{f}-t_{0}}$

Normalized Jerk $=\frac{\mathrm{d} X^{3}}{\mathrm{~d}^{3} T}$

Normalized Minimum Jerk, $C_{\text {Normalized_Jerk }}=\int_{t_{0}}^{t_{f}}\left\{\frac{\mathrm{d} X^{3}}{\mathrm{~d}^{3} T}\right\}^{2} \mathrm{~d} T$

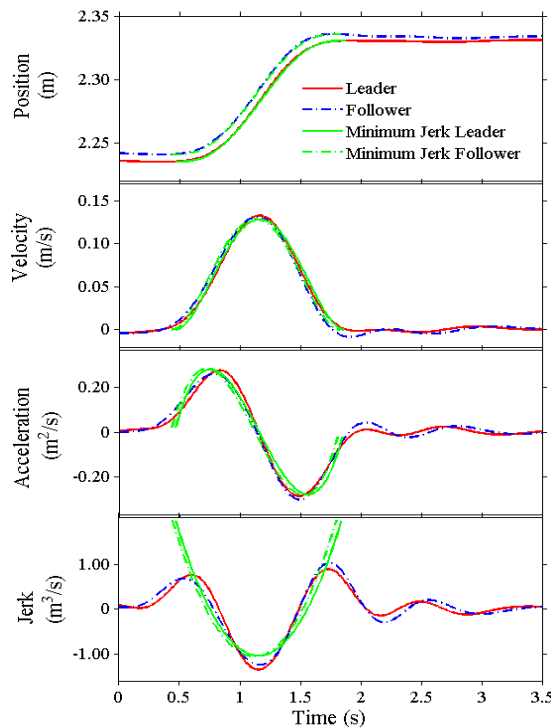

(a)Profiles of a smooth leftward/rightward cooperative task.

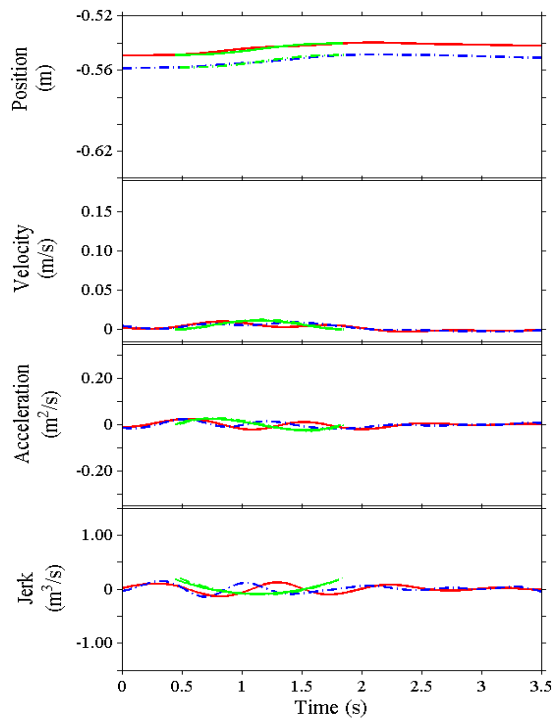

(c) Profiles calculated in the direction perpendicular (upward/downward) to cooperative task in (a).

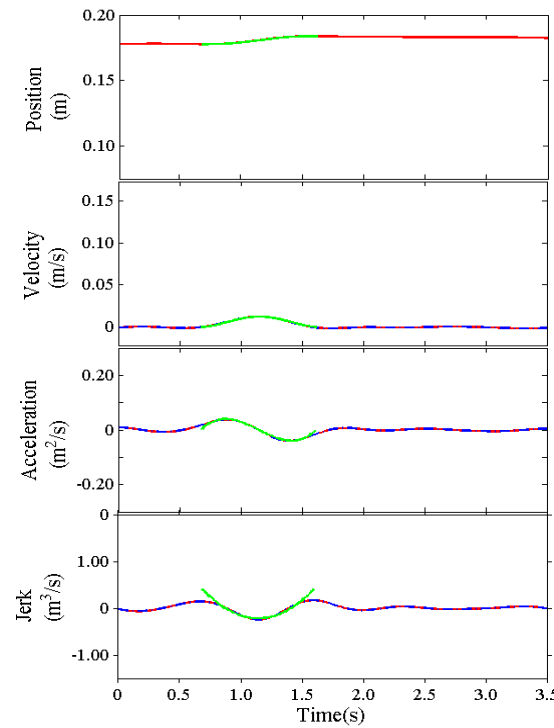

(b) Profiles calculated in the direction perpendicular (forward/backward) to cooperative task in (a).

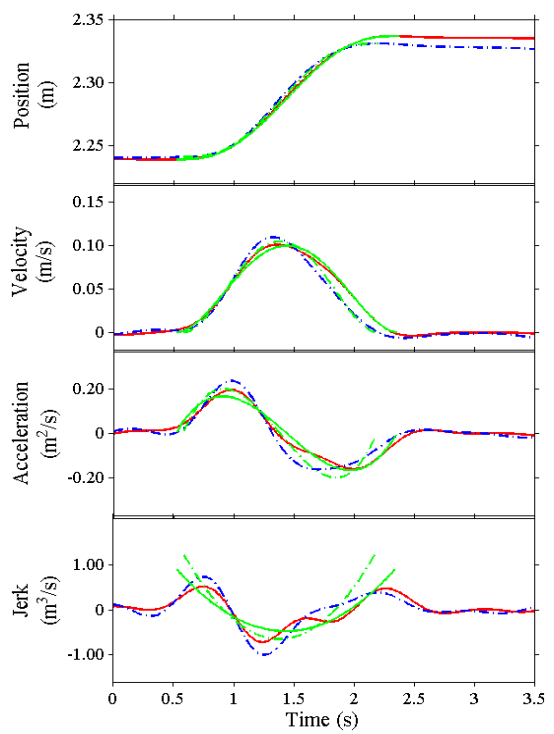

(d) Profile of an awkward leftward/rightward cooperative task.

Fig. 4 Profiles of position, velocity, acceleration and jerk during cooperative task 


\subsection{Smoothness versus modes of information delivery to the Follower}

Figure 5 shows the motion's smoothness with regards to the cooperative task in leftward/rightward, upward/downward and forward/backward direction over the aforementioned four modes (refer to Table 1) of information delivery for End and Center cases, respectively.

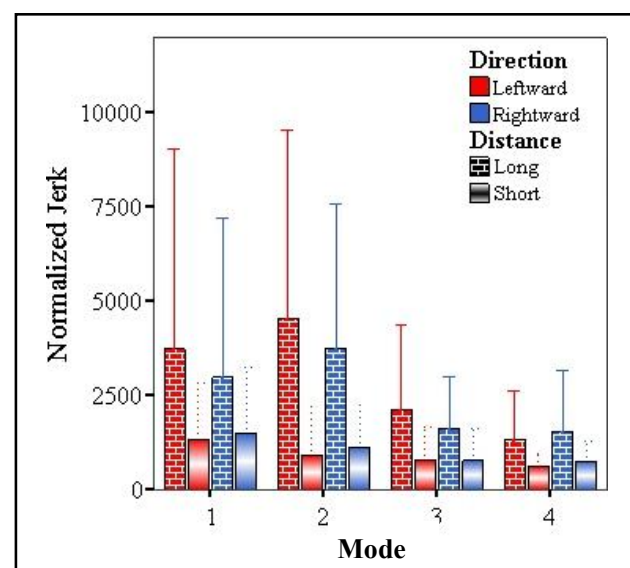

(a) Leftward/rightward (End)

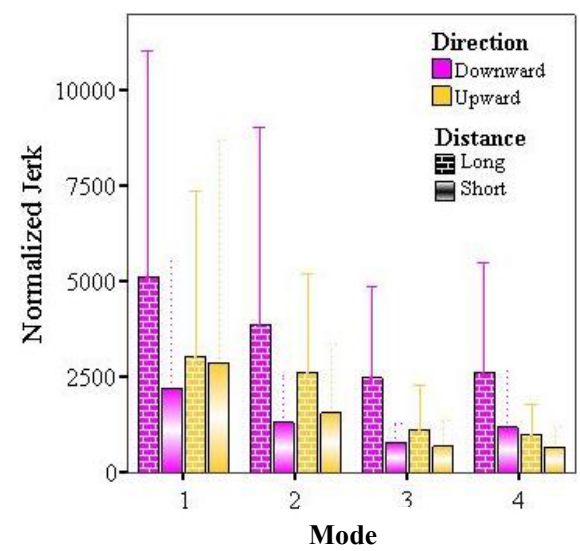

(c) Upward/downward (End)

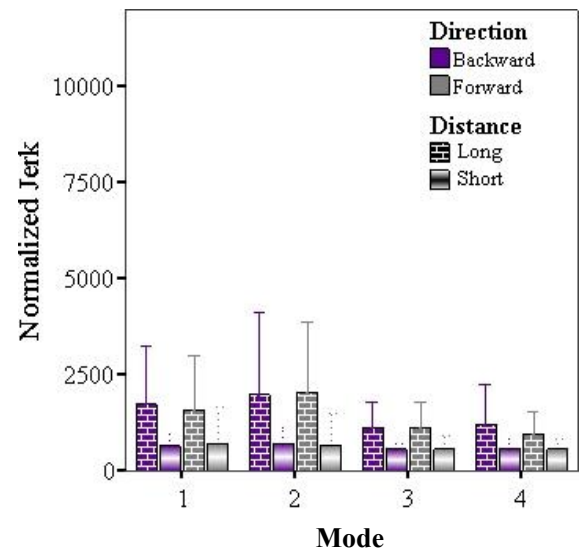

(e) Forward/backward (End)

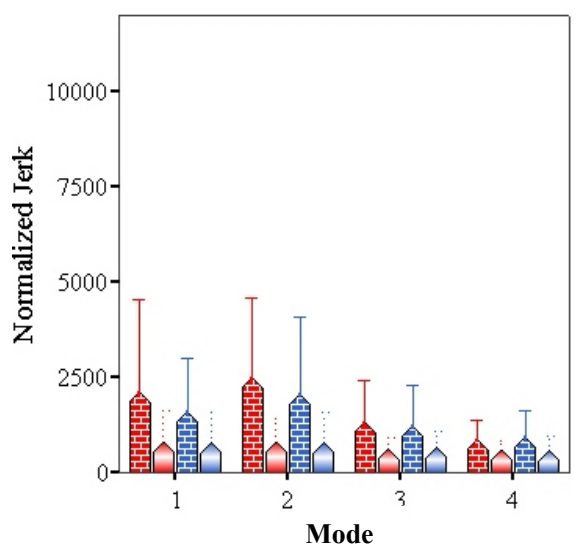

(b) Leftward/rightward (Center)

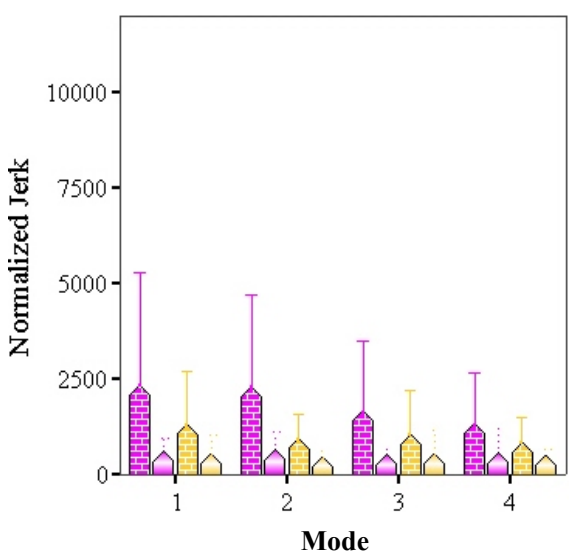

(d) Upward/downward (Center)

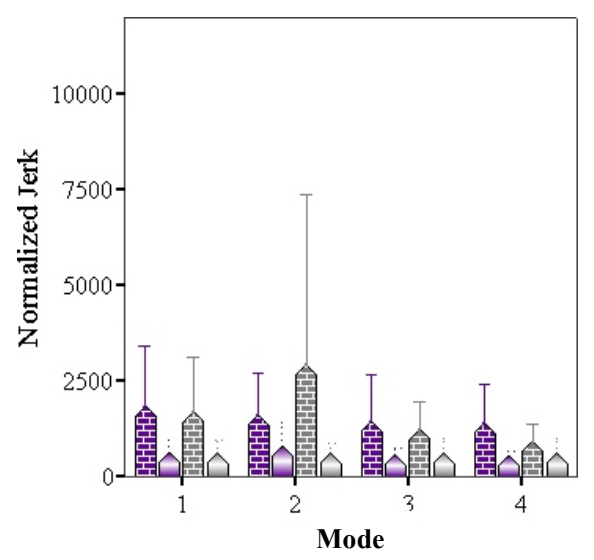

(f)Forward/backward (Center)

Fig. 5 Smoothness over the mode of information delivery for the cooperative task in each direction for End (Left column) and Center (Right column) case.

Each bar in each mode represents the center point (a center point on the object representing both subjects motion) average normalized jerk of 10 repetitions of all groups' motions. These repetitions consist of a same direction and distance variables combinations. In each mode, the first bar indicates the long distance with a first direction (which refers to 
the order of the direction in each graph, i.e. in leftward/rightward direction, the first direction is leftward, in upward/downward is downward, and in forward/backward is backward) of the cooperative task, and the second bar shows the short distance with the first direction of the cooperative task. Subsequently, the third and fourth bars represent the cooperative task in long and short distance with a second direction (which refers to rightward in leftward/rightward, upward in upward/downward, and forward in forward/backward), respectively. The error bars indicate the standard deviation of the normalized jerk. A smaller mean normalized jerk value indicates a smooth cooperative motion and vice versa. The distance traveled in each cooperative direction is within the range mentioned in $\S 3.2$ and is shown in Fig. 6. As mentioned in $\S 1$, the precise positioning phase was excluded and subjects stopped the object at the vicinity of the target area. Also, it was verified that the variation of distance within and between the cooperative task direction

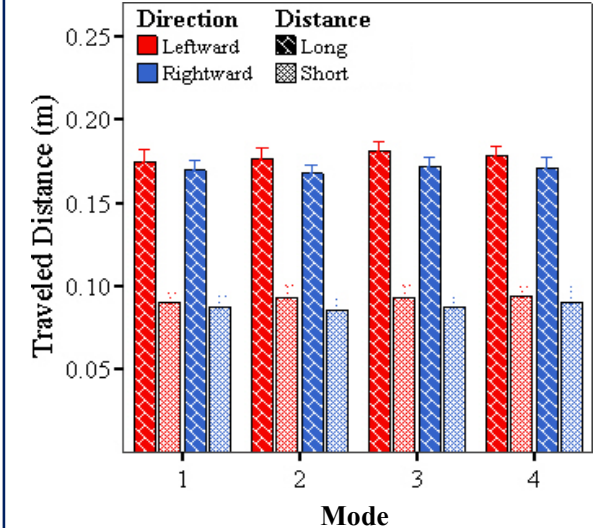

(a) Leftward/rightward (End)

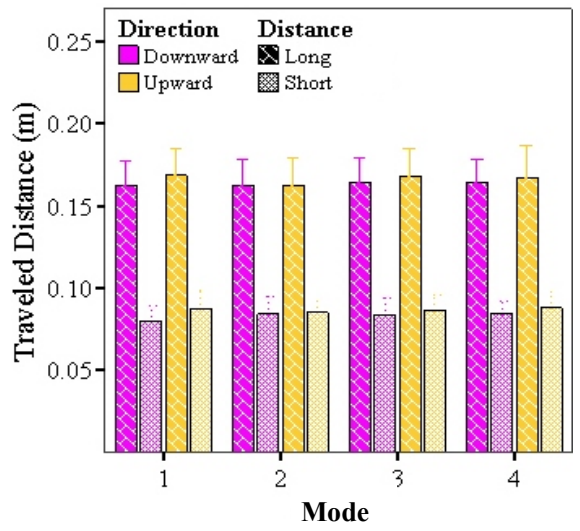

(c) Upward/downward (End)

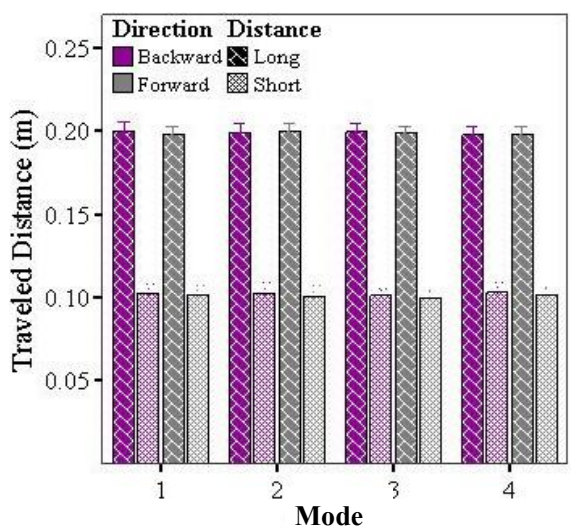

(e) Forward/backward (End)

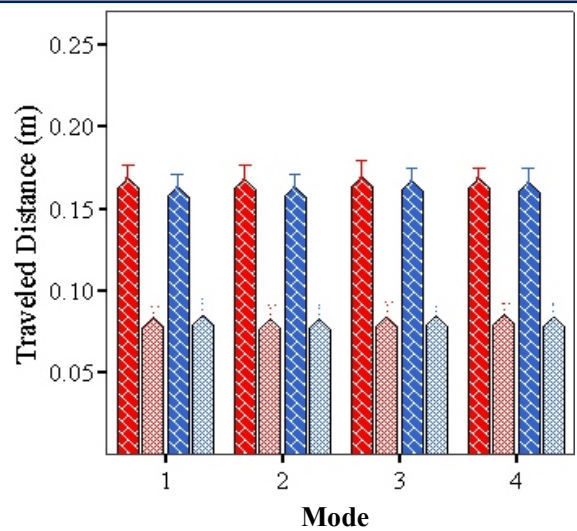

(b) Leftward/rightward (Center)

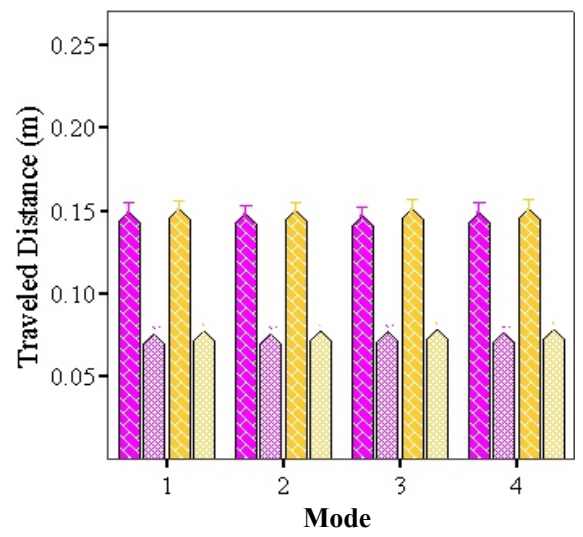

(d) Upward/downward (Center)

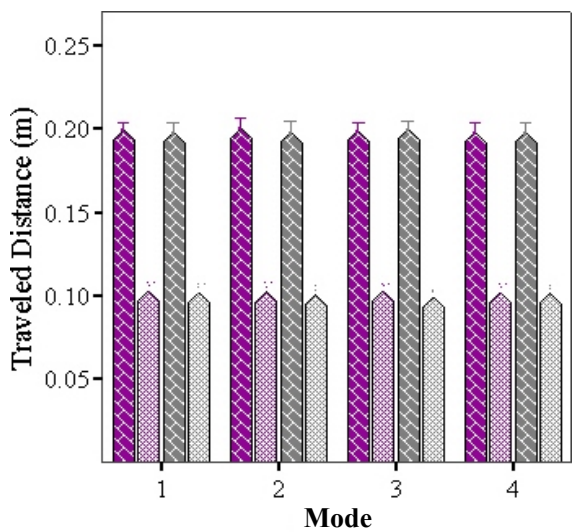

(f) Forward/backward (Center)

Fig. 6 Traveled distance in each cooperative task direction. 
did not influence the smoothness indicated by mean normalized jerk.

The effect of mode on the mean normalized jerk was investigated. Mauchly's test indicated that the assumption of sphericity had been violated for the main effects of mode in each direction (leftward/rightward, $\chi^{2}(5)=35.76$; upward/downward, $\chi^{2}(5)=45.51$; forward/backward, $\chi^{2}(5)=30.15$, all $\left.p \mathrm{~s}<0.01\right)$, therefore degrees of freedom were corrected using Greenhouse Geisser estimates of sphericity (leftward/rightward, $\varepsilon=0.745$; upward/downward, $\varepsilon=0.586$; forward/backward, $\varepsilon=0.723$ ). The result shows that there was a significant effect of modes on mean normalized jerk in each direction (leftward/rightward, F $(2.23,100.5)=59.81$, upward/downward, F $(1.76,79.10)=24.09$, forward/backward, F $(3,97.6)=23.64)$, all $p$ s $<0.01)$. Obviously, regardless of the cooperative task directions, the mean normalized jerk value reduces towards mode 4 and furthermore, this mode is associated with the least standard deviation.

Thus, regardless of which part of the image was being perceived, if a human has explicit information on the target destination and signals to initiate motion, a natural and smooth cooperative motion was frequently generated. Also it shows that the explicit information was more dominant than the implicit information (following the Leader motion using implicit information did not generate more frequent smoother cooperative task, e.g. mode 1) for the Follower to predict the Leader's intention on start and stop motion. Therefore, for a smooth human-robot cooperation (robot as the Follower), both information must be made available to the robot. The effect of distance on mean normalized jerk was found significant in all directions (leftward/rightward, $F(1,45)=282.25$, upward/downward, $\mathrm{F}(1,45)=142.58$, forward/backward, $\mathrm{F}(1,45)=403.87$, all $p \mathrm{~s}<0.01)$. This means that the long distance cooperative motion generates a higher mean jerk and standard deviation compared to the short distance motion. The higher standard deviation in each bar shows that, for 10 trials of motion, some generate smooth whilst some produce awkward cooperative motion. The difference of cooperative task smoothness between sub-direction (e.g. between leftward and rightward in leftward/rightward cooperative task) was shown in the graph. In leftward/rightward direction and forward/backward direction, the statistical test performed indicated that the differences were not significant, $\mathrm{F}(1,45)=$ $2.19, p>0.01$ and $\mathrm{F}(1,45)=0.63, p>0.01$, respectively. However, the statistical test performed for upward/downward direction shows significant difference between its sub-direction, $\mathrm{F}(1,45)=35.53, p<0.01$. Leftward and rightward direction task smoothness was similar because the task was not associated with a large change in gravitational force ${ }^{(8)}$. In forward/backward, forward was mentioned to be more awkward than backward ${ }^{(8)}$. The reason is that the Follower has to support more weight when he extended his hand during the task. However, in forward/backward task, the effect of hand extension should be cancelled out by both subjects (in Forward task, Leader extended, the Follower flexed; in Backward task, Leader flexed and Follower extended). Thus, the similarity of task smoothness should exist between the sub-direction. The difference in results with the previous researcher may also exist due to the use of the jerk value rather than the error value $^{(8)}$ as smoothness indicator. In upward/downward, the downward task was associated with more awkward task ${ }^{(8)}$. Subjects informed that they have to be more careful during the downward task, since they feel lighter when moved the object from start to target position. Analysis on average velocity indicated that during lower average velocity was observed during downward compare to upward task.

The difference between End and Center case was tested for each task direction, e.g. leftward/rightward End case was compared with leftward/rightward Center case; the same procedure was carried out for upward/downward and forward/backward cooperative task. A significant difference was found between the End and Center cases for the cooperative task in leftward/rightward, $\mathrm{F}(1,45)=69.62, p<0.01$, and upward/downward direction, $\mathrm{F}(1$, $45)=114.57, p<0.01$. This means that the Center case generates a smoother cooperative 
motion. However, the same effect was not observed for the cooperative task in the forward/backward direction, $\mathrm{F}(1,45)=1.30, p>0.01$. In the Center case of leftward/rightward and upward/downward cooperative task, both subjects looked at and shared almost the same area of the experimental object. Thus, both subjects managed to visualize and share the same information of the object's motion and moved the same part of the object in the same axis and plane towards target position (see Fig. 7(a)). Eventually, it produced more frequent, smoother and natural cooperative motion.

In contrast, during the End case of leftward/rightward and upward/downward cooperative task, both subjects looked at the end part of the 2D image. Thus, it was similar for them to look at the experimental object's end part that was closed to their hands. Since the image has two ends, therefore, both subjects were not looking at the same area while moving and matching the experimental object to the target position. Thus, both subjects did not visualize and share the same information about the object's motion and they moved the object in a separate axis and plane towards target position (see Fig. 7(b)). In human-robot cooperation, therefore, it is suggested that both subjects should visualize and share the same information of the object's motion to generate a smooth cooperative motion.

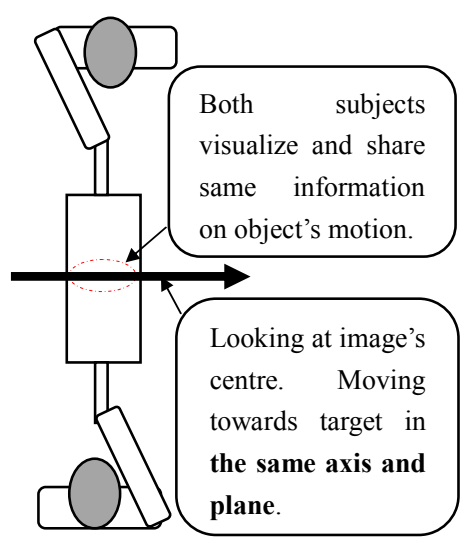

(a) Center case

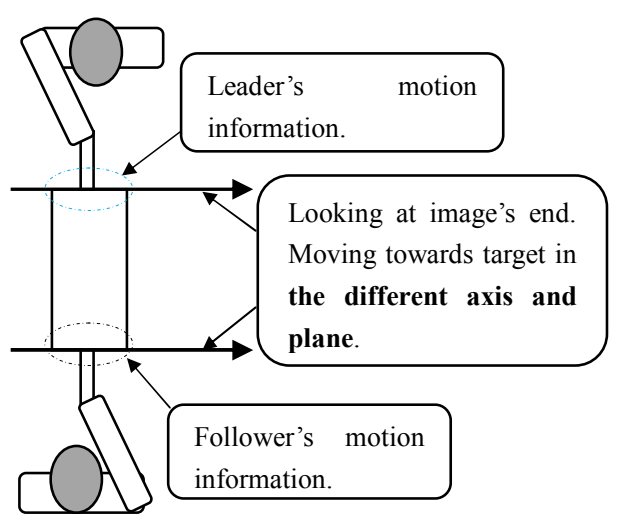

(b) End case

Fig. 7 The difference in visualizing and sharing the information between Center and End case in leftward/rightward and upward/downward cooperative task. Arrows indicates the direction of the cooperative motion. In Center case, both subjects share the same information and the arrow is thicker (to show sharing of information). In End case, they move the image based on the separate object's motion information.

Figure 8 and 9 show different characteristics between the Center and End cases of the cooperative task in leftward/rightward direction, respectively. The example was taken from the same group of subjects, with the same distance and direction. In Fig. 8 (Center case), the position profiles of both subjects are almost similar from the beginning towards the target position. They also managed to reach the target position at the same time, as shown in Fig. 8(b). In contrast, in Fig. 9 (End case), they did not look at the same part of the image; the position profile of both subjects started to be apart when time approached between 0.5 and 1 s. Eventually, the cooperative motion became awkward. Moreover, they did not reach the target position at the same time, as shown in Fig. 9(b) (see velocity profile, the Leader reached zero velocity faster than the Follower). The phenomena shown in figure 8 and 9 were also observed in upward/downward direction. However, in forward/backward direction task, regardless of End or Center, both subjects managed to reach the target at the same time. 
a)

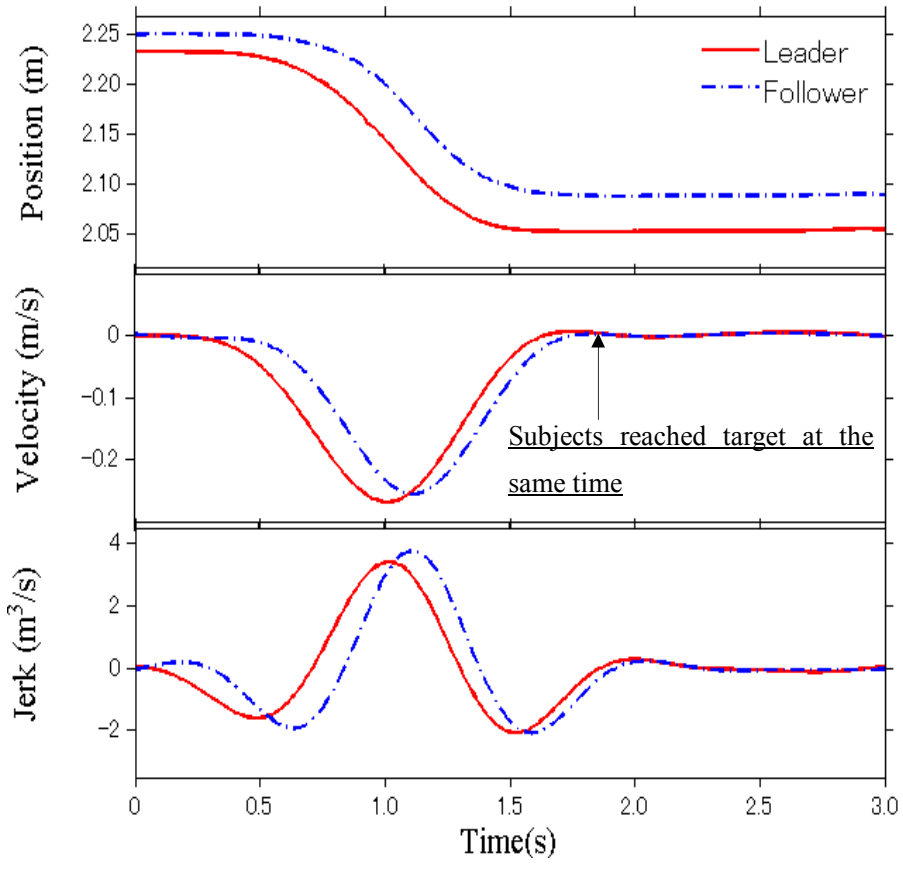

Fig. 8 Trajectories of cooperative task for Center case in leftward/rightward direction. From top: a). Actual position in the direction of cooperative motion. b). Actual velocity profile. c). Actual jerk profile.

a)

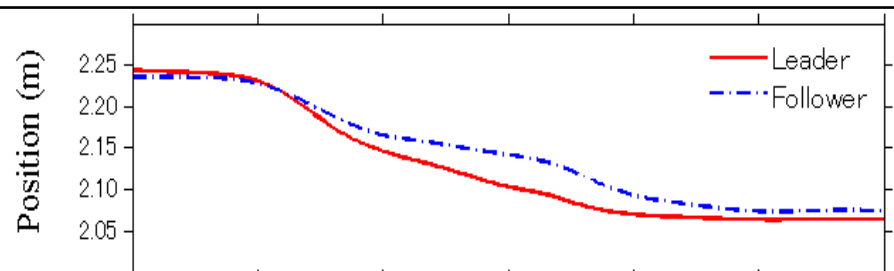

b)

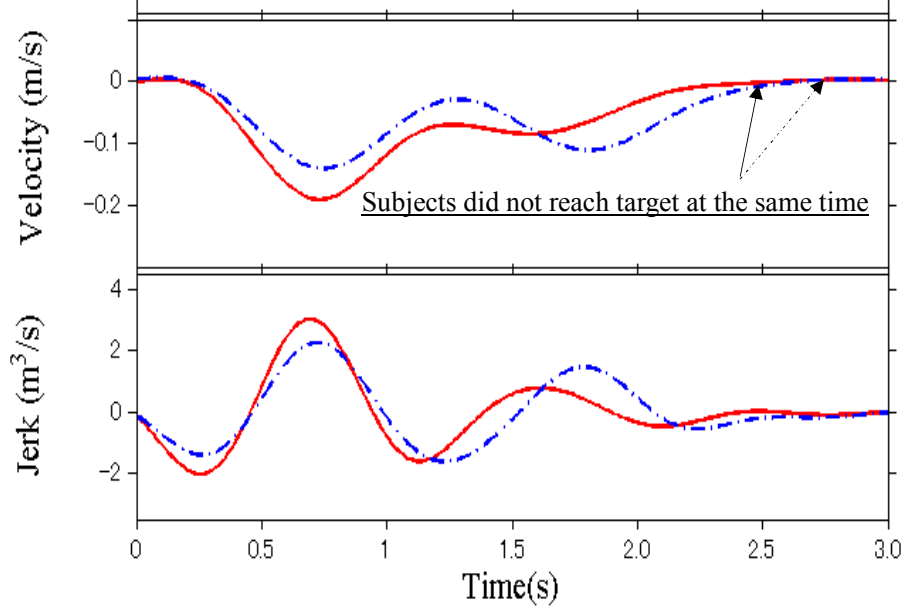

Fig. 9 Trajectories of cooperative task for End case in leftward/rightward direction. From top: a). Actual position in the direction of cooperative motion. b). Actual velocity profile. c). Actual jerk profile.

The cooperative task in forward/backward direction was reported to being more influenced from the force interaction (implicit information) between the Leader and the Follower (11)-(16). Relative to the cooperative task in leftward/rightward and upward/downward direction, the smoothness of the task in forward/backward in mode 1 is almost similar to mode 4, as shown in Fig. 5(e) and 5(f). In mode 1, the Follower was not provided with any explicit task information; he/she depends on implicit information to estimate a task's initiation and termination. Thus, our experimental results also indicate that in forward/backward cooperative task; force interaction is more influential on the cooperative motion smoothness. Therefore, regardless of looking at End or Center, the force 
interaction between subjects generates similar cooperative motion smoothness.

Another thought for the similarity between Center and End case in forward/backward direction is due to the information that they share during the cooperative task. In Center case, they shared the same information of the object and moved in the same axis and plane (see Fig. 10(a)) as in leftward/rightward and upward/downward Center case. However, for the End case, although they did not visualize the same part of the object, they still share the same object's motion since they move the object in the same axis and plane towards the target position (see Fig. 10(b)). Perhaps, this also another factor that has induced the similarity between End and Center cases for the cooperative task in forward/backward direction.

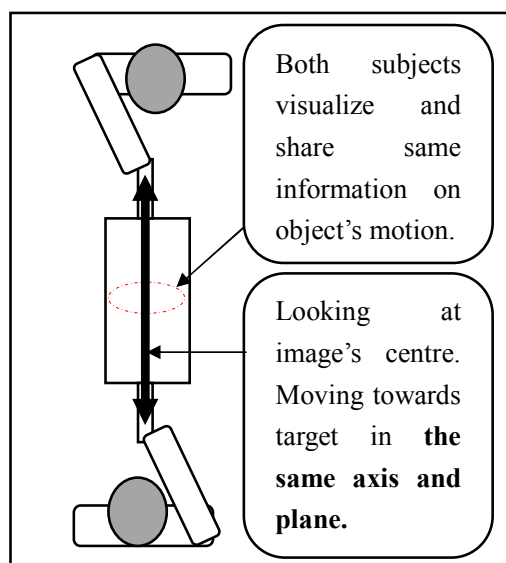

(a) Center case

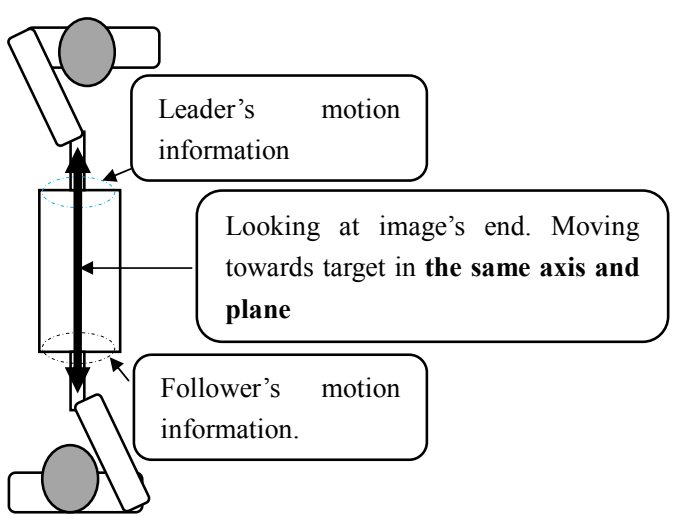

(b) End case

Fig. 10 Visualizing and sharing the same information for Center and End case in forward/backward direction cooperative task. Arrows indicates the direction of the cooperative motion.

\subsection{Object Rotational Motion and Cooperative Task Smoothness}

The following figure shows the relationship between object rotational motion (average angle) and cooperative task smoothness. The object's angle was calculated on the plane parallel to the cooperative task direction (e.g. leftward/rightward task was executed parallel to horizontal plane, thus the angle was calculated on based on horizontal plane). We hypothesized that less object rotation was generated during smooth cooperative motion. Thus, the results presented were obtained from mode 4 where more frequent and smooth task was generated. The result shows that smooth tasks (Normalized jerk value is less than 600 ) in End case were associated with a range of 0 to 8 degrees angular change during the task. On the other hand, the smooth tasks in Center case were associated with lower range of angular change, i.e. 0 to 5 degrees. Thus, we could infer that smooth tasks in Center case were associated with less object rotation compare to the smooth task in End case. The result is true for leftward/rightward and upward/downward direction. However, in forward backward direction, almost similar range of angular change was observed either in End or Center case. The effect of End or Center is not significant on the object rotation in smooth cooperative task of forward/backward direction. 


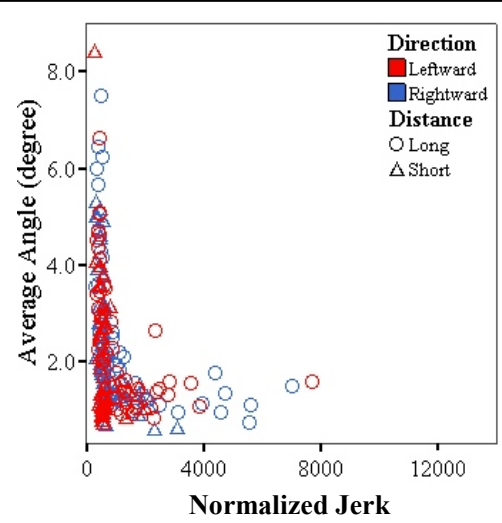

(a) Leftward/rightward (End)

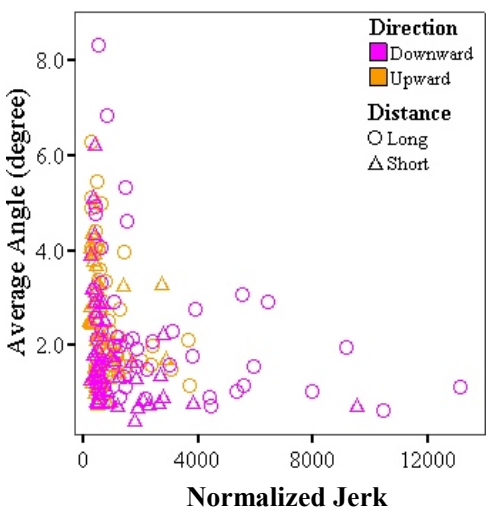

(c) Upward/downward (End)

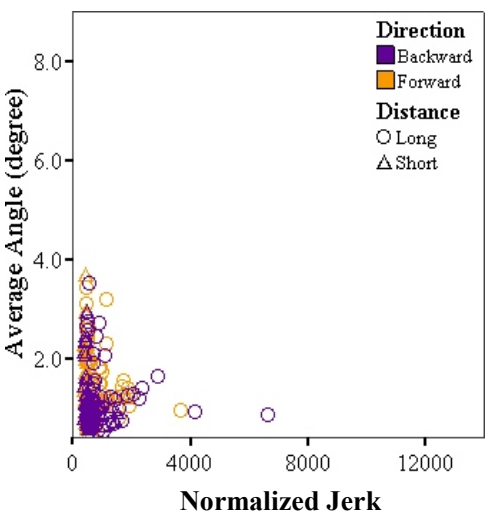

(e) Forward/backward (End)

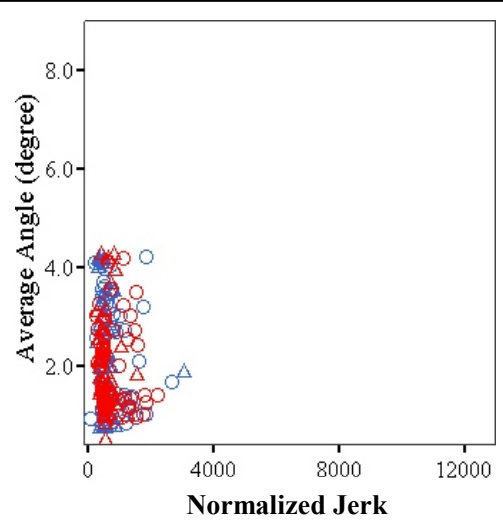

(b) Leftward/rightward (Center)

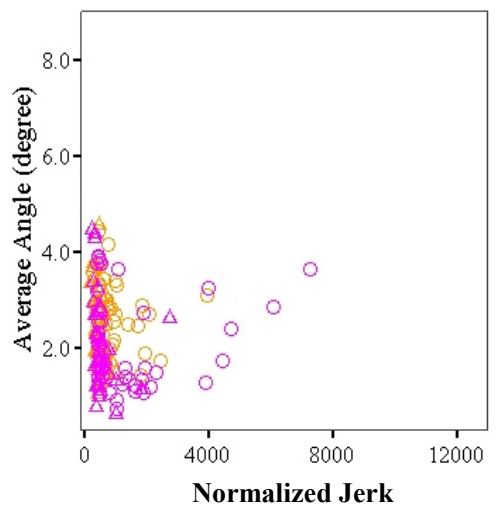

(d) Upward/downward (Center)

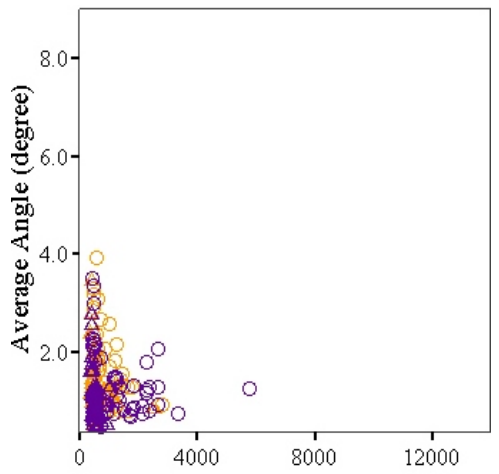

Normalized Jerk

(f) Forward/backward (Center)

Fig. 11 Object rotational motion over cooperative task smoothness in each task direction for End (Left column) and Center (Right column).

\section{Conclusion}

We are aiming at developing a smooth cooperative object transfer in human-robot system mimicking the smoothness of the task executed by two humans. Prior to the development of such system, we have investigated the characteristics for generating a smooth cooperative object transfers in human-human system with regards to the information exchanges occurs between subjects. The analysis revealed that explicit information induced more frequent smooth cooperative task regardless of which part of the object is being perceived during the task. The result is in agreement with the previous research ${ }^{(8),(17)}$. Comparing either End or Center case, the result shows that the Center case (both subjects perceived at same area on the object) generated more frequent, smoother and natural cooperative motion. Moreover, the Center case shows less objects rotation during 
smooth cooperative task. The result is true for the cooperative task executed in leftward/rightward and upward/downward direction; however, it is not for the cooperative task executed in forward/backward direction. In forward/backward direction, the task smoothness did not show any significant difference between the End and Center case. Also, in both End and Center cases, a similar range of object rotation during smooth task was observed. A force interaction between subjects is more influential on the cooperative task smoothness than visual effect in forward/backward direction.

It was understood from the previous and current research that in mode 4, the Follower did not follow the Leader motion from the beginning ${ }^{(8)}$, however, he/she generated smooth trajectory towards the target position based on his/her own motion. In implementing mode 4 in human-robot system, where robot acts as Follower, the robot is not required to follow the human Leader to generate a smooth cooperative task (similar to the phenomenon in human-human system). Once received instruction to initiate the task, the robot could move towards the target with its own rhythm. In other words, the Leader motion is not required to be feedback to the robot to generate a smooth cooperative task. Thus, the robot will be adapted with a smooth trajectory of the human Follower which was obtained from mode 4 of human-human cooperative system. The smooth trajectory agrees with the trajectory generated using the Minimum Jerk Model. Thus, the trajectory of the robot Follower will be based on the trajectory of the Minimum Jerk Model. In using the Minimum Jerk Model to generate a smooth trajectory for the robot Follower mimicking the trajectory of human Follower (human-human system), the relationship between movement time and travel distance of human Follower is required. Another result obtained in this research and to be implemented in human-robot system is the Center case. Therefore, based on the Centre case, the relationship of movement time and travel distance of the Follower (human-human cooperative system) will be investigated and adapted to the robot motion (human-robot cooperative system). Although the effect of End and Center case may be significant in human-human system; however, in human-robot system, both cases should be further tested and evaluated.

For future works, consideration on a longer target's distance (short $>100 \mathrm{~mm}$ and long $>200 \mathrm{~mm}$ ), wider range of subject age (between 25 to 65 years of working age) ${ }^{(9)}$ and perhaps, on the variation of object's weight ${ }^{(20)}$ should also be given to mimics the real cooperative transfer between humans.

\section{References}

(1) Pramilia Rani, Jared Sims, Robert Brackin, Nilanjan Sarkar, Online Stress Detection using Psychophysiological Signal for Implicit Human-Robot Cooperation, Robotica, Vol. 20, No.6(2002), pp.673-686.

(2) Omar M. Al Jarrah, Yuan F. Zheng, Arm manipulator coordination for load sharing using compliant control, Proceedings of the of International Conference on Robotics and Automation, (1996-4), pp.1000-1005.

(3) Y. Aiyama, M. Hara, T. Yabuki, J. Ota, T. Arai, Cooperative transportation by two four-legged robot with implicit communication, Robotics and Autonomous Systems, Vol. 29, No.1(1999), pp.13-19.

(4) Xin Yang, Keigo Watanabe, Kiyotaka Izumi, Kazuo Kiguchi, A Common Reference Object Concept to Cooperative Transportation, Proceedings of the IEEE International Conference on Robotics and Automation, (2003-5), pp.515-520.

(5) Markus Huber, Markus Rickert, Alois Knoll, Thomas Brandt, Stefan Glasauer, Human-Robot Interaction in Hand-Over Task, Proceedings of the IEEE International Symposium on Robot and Human Interactive Communication, (2008-8), pp.107-112.

(6) Yasuo Hayashibara, Yukinobu Sonoda, Tomohiko Takubo, Hirohiko Arai, Kazuo 
Tanie, Assist System for Carrying a Long Object with a Human - Analysis of a human cooperative behavior in the vertical direction, Proceedings of the IEEE International Conference on Intelligent Robot and Systems, (1999-10), pp.695-700.

(7) Sylvain Miossec, Abderrahmane Kheddar, Human Motion in Cooperative Transfer: Moving Object Case Study, Proceedings of the IEEE International Conference on Robotics and Biomimetics, (2009-2), pp.1509-1514.

(8) Shahriman Abu Bakar, Ryojun Ikeura, Yuichiro Handa, Takemi Yano, Kazuki Mizutani, Hideki Sawai, Communication during the Cooperative Motion in the Task of carrying an object between two humans, Journal of Biomechanical Science and Engineering, Vol. 5, No.2(2010), pp.104-118.

(9) Brach Poston, Arend W.A. Van Gemmert, Beth Barduson, George E.Stelmach, Movement structure in young and elderly adults during goal-directed movements of the left and right arm, Brain and Cognition, Vol. 69, No.1(2009), pp.30-38.

(10) T. Flash, N. Hogan, The coordination of the arm movement: an experimentally confirmed mathematical model, Journal of neuroscience, Vol.5, No. 7(1985), pp. 1688-1703.

(11) P. M. Fitts, The information capacity of the human motor system in controlling the amplitude of movement, Journal of Experimental Psychology, Vol. 121, No. 3(1954), pp.262-269.

(12) Ikeura, R., Inooka, H., \& Mizutani, K., Subjective evaluation for maneuverability of a robot cooperating with humans. Journal of Robotics and Mechatronics, Vol.14, No.5 (2002), pp.324-329.

(13) Md. Mozasser Rahman, Ryojun Ikeura, Kazuki Mizutani, Cooperation Characteristics of Two Humans in Moving an Object, Journal of Machine Intelligence \& Robotic Control, Vol.4, No.2(2002), pp.43-48.

(14) Ryojun Ikeura, Hiroshi Monden, Hikaru Inooka, Cooperative Motion Control of a robot and a human, Proceedings of the IEEE International Workshop on Robot and Human Communication, (1994-7), pp.112-117.

(15) M.M. Rahman, R. Ikeura, K. Mizutani, Analysis of Cooperation characteristics of two humans in moving an object, Proceedings of the International Conference on Mechatronics and Information Technology, (2001-12), pp.454-458

(16) Ryojun Ikeura, Tomoki Moriguchi, Kazuki Mizutani, Optimal Variable Damping Control for a Robot Carrying an Object with a Human, Proceedings of the International Conference on Control, Automation and Systems, (2001-10), pp.63-66.

(17) Shahriman A.B, R. Ikeura, Ahmad Faizal Salleh, T. Yano, K. Mizutani and Hideki Sawai, A Study of Human-Human Cooperative Characteristic in Moving an Object, Proceedings of the IEEE International Conference on Human System Interaction, (2009-5), pp.679-684.

(18) Ahmad Faizal Salleh, Ryojun Ikeura, Shahriman Abu Bakar, Takemi Yano, A motion analysis of humans performing cooperative task in Forward/backward direction, Proceedings of the SPIE, Vol. 7500, (2009), 75000Y-1.

(19) Ahmad Faizal Salleh, Ryojun Ikeura, Soichiro Hayakawa, Hideki Sawai, Towards human-robot cooperative object transfer: Perceiving different part of the object during task and motion smoothness, Proceedings of the IEEE International Conference on Robotics and Biomimetics, (2010-12), pp.549-554.

(20) Ruud G. J. Meulenbroek, Jurjen Bosga, Majken Hulstin, Stephan Miedl, Joint action coordination in transferring objects, Experimental Brain Research, Vol. 180, No. 2(2007), pp.333-343.

(21) Tytus Wojtaraa, Masafumi Uchihara, Hideyuki Murayama, Shingo Shimoda, Satoshi Sakai, Hideo Fujimoto, Hidenori Kimura, Human-robot collaboration in precise positioning of a three-dimensional object, Automatica,Vol.45, No.2(2009), 
pp.333-342.

(22) Milner, T. E., A model for the generation of movements requiring endpoint precision, Neuroscience, Vol. 49, No.2(1992), pp.487-496.

(23) Sara Kiesler, Pamela Hinds, Introduction to this Special Issue on Human-Robot Interaction, Human Computer Interaction, Vol.19, No.1(2004), pp.1-8. 